\title{
Focus Control in HIFU Therapy Assisted by Time-Reversal Simulation with an Iterative Procedure for Hot Spot Elimination*
}

\author{
Nicolas LEDUC ${ }^{* *}$, Kohei OKITA***, Kazuyasu SUGIYAMA**, Shu TAKAGI**, \\ and Yoichiro MATSUMOTO** \\ ** Department of Mechanical Engineering, School of Engineering, The University of Tokyo \\ 7-3-1 Hongo, Bunkyo-ku, Tokyo, 113-8656, Japan \\ E-mail: sugiyama@fel.t.u-tokyo.ac.jp \\ *** Department of Mechanical Engineering, College of Industrial Technology, Nihon University \\ 1-2-1 Izumi-cho, Narashino, Chiba, 275-8575, Japan \\ †rgan and Body Scale Team, RIKEN \\ 2-1 Hirosawa, Wako, Saitama, 351-0198, Japan
}

\begin{abstract}
Both focus control and hot spot elimination applied to the High Intensity Focused Ultrasound (HIFU) treatment are addressed using three-dimensional numerical simulations, which can faithfully capture the pressure field in the HIFU system involving strong heterogeneity in material properties. The hot spot corresponds to the superficial secondary pressure peak, which originates in the reflection and refraction of the ultrasonic waves. As an extension of the Time Reversal theory (Fink, 1992, IEEE Trans. Ultrason. Ferroelectr. Freq. Control, 39, 555), which is based on the reversibility of the linear wave equation, a heuristic, iterative and self-adaptive process is proposed to make the HIFU system mounting array piezotransducers dynamically controllable. The amplitude and phase of the driving pressure on each transducer are determined from the temporal change in the monitored pressure of diverging acoustic waves emitted from virtual acoustic sources at the hot spot as well as the target point. The primary peak is found to be strengthened through the process, while the secondary peaks are to be obliterated. The present method is applied to a transskull HIFU simulation using human body voxel data, and its effectiveness is discussed.
\end{abstract}

Key words : HIFU, Time Reversal, Grating Lobes, Secondary Hot Spots

\section{Introduction}

\subsection{Focus control in HIFU through heterogeneous media}

Although nowadays widely spread for imaging and treatments uses, High Intensity Focused Ultrasounds (HIFU) techniques are still limited by two main factors. One is a nonlinearity in a density-pressure relationship, which brings a nonuniform speed of sound in the wave propagation. The other is a material heterogeneity inside the human body, which causes refraction and reflection. Both the factors are likely to make the wave pattern complicated. As illustrated in Fig. 1, seriously, a focal point in the pressure field is displaced from a target located at the geometric focus of a piezotransducer surface when the sound wave is pulsed from the piezotransducer array with the same phase. Particularly in the case of therapeutic ultrasound applications such as lithotripsy and tumor heating, precise control of the pressure pattern is required to enhance the heating effect within the aimed area. It is obviously critical to avoid overheating normal brain and tissues. Improvement of current HIFU techniques as well as the development of new fields of application will require better knowledge of focus control. Diverse possible solutions have been tried such as increasing the angular aperture

${ }^{*}$ Received 1 Nov., 2011 (No. 11-0664) [DOI: 10.1299/jbse.7.43]

Copyright (c) 2012 by JSME 


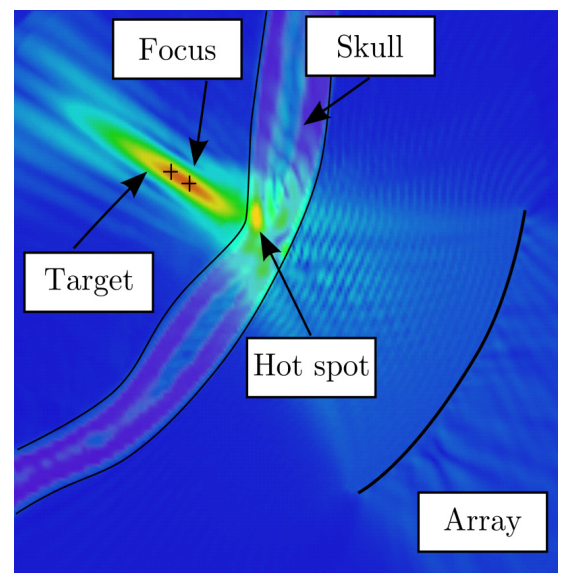

Fig. 1 A cross-sectional view of a typical pressure field of transskull HIFU simulations at a driving frequency of $f=500 \mathrm{kHz}$. The skull has a density of $\rho=1700$ $\mathrm{kg} / \mathrm{m}^{3}$ and a bulk modulus of $K=10 \mathrm{GPa}$. The color scale display of the pressure exhibits frontal lobes from a piezotransducer with $6.5 \mathrm{~cm}$ aperture. The heterogeneity in the propagation media results in a displacement between the targeted and focal points. Further, a hot spot, corresponding to a secondary pressure peak inside the skull, is generated thereby.

of the piezo array, using low absorption frequencies, simplified projective models for heterogeneity $^{(1)}$, or performing time-shifting techniques such as the procedures developed by Sun and Hynynen ${ }^{(2),(3)}$. Those methods led to promising results, but time-shifting predictions remain difficult, and physical limits restrain the angle of piezotransducer arrays. A method has risen as a possible solution to the focus control problem: the so-called Time Reversal (TR) theory ${ }^{(4),(5)}$ can be used to control the focus of the ultrasound with seemingly unchallenged precision. The TR theory specifies that solutions to the linear wave equation

$$
\left(L_{r}+L_{t}\right) p=0
$$

where $L_{r}=\nabla \cdot\left\{(\rho(\boldsymbol{r}))^{-1} \nabla\right\}$ and $L_{t}=\chi(\boldsymbol{r}) \partial_{t}^{2}$, usual function operators of the wave equation, are invariant with respect to the transformation $t \Leftrightarrow-t$. This property leads the way to the description of self-adaptive procedures, independent from the propagation media, and fully non-invasive HIFU treatments. So far, the applicability of the TR scheme has been demonstrated by Fink and co-workers. The TR cavity was theorized ${ }^{(6)}$ and TR mirrors were fully experimented ${ }^{(7)}$. Then, Aubry et al. ${ }^{(8)}$ and later Marquet et al. ${ }^{(9)}$ achieved the combination of 3D CT scan simulation and experiment to successfully perform transcranial focus control of ultrasound waves through the skull of a monkey, leading to promising results in focus control of HIFU propagation through strongly heterogeneous media.

\subsection{Secondary peaks issues}

While the time-shifting and TR methods provide remarkable improvement of focus location, a remaining issue in the field of phased-array induced focus control is the generation of parasite secondary intensity peaks close to either i) areas of constructive interference of the pressure fields generated by the ultrasonic devices, or ii) strongly heterogeneous structures of the propagation media, such as skull bones inducing hot spots as illustrated in Fig. 1. Those two phenomena are not exclusive and can entail negative consequences in the HIFU treatment.

i) On one hand are the constructive diffraction patterns essential to the discrete nature of phased arrays, and common to the whole domain of arrays physics, with, for instance, a whole body of publications in the sonar and radar fields ${ }^{(10)}$. In the ultrasonic sciences, this factor has been addressed in various ways, most of them attempting physical modifications of the piezotransducers: see the structural filtering of supercritical plates ${ }^{(11)}$, or the modification of the piezotransducers shape ${ }^{(12)}$. Differently, electrical or electronic methods for limiting 
secondary foci have been tested, such as modifying the energy of each piezotransducer ${ }^{(13)}$, or filtering and modulating the generated signal ${ }^{(14)}$. However, the seemingly most successful methods appeal to randomization of the piezotransducer location and shape on the phased array surface ${ }^{(15),(16)}$.

ii) On the other hand is a non-negligible increase of the intensity of the secondary peaks associated with the presence of strong heterogeneity in the propagation media. Increase of the local sound speed leads to a premature focus of the sound waves in the high-speed areas, and therefore unexpected concentration of acoustic energy next to the treatment area surface, that is often addressed by means of cooling devices. This relatively high value of secondary peak intensity over the main peak maximal pressure $P$ is also vastly dependent on the aperture of spherically shaped phased arrays, and the distance between the target and the interface of the heterogeneous structure. Large aperture arrays are known to limit diffraction effects and improve deposition of energy on the central area. In the same way, parasite energy peaks are comparatively decreased. The effects on focus location, device aperture and signal frequency over the apparition of secondary hot spots can be inferred from numerical results by Sun and Hynynen ${ }^{(3)}$. They performed a phaseoptimizing simulation with a device fully surrounding the skull using a low density and speed model for the skull $\left(\rho=1796 \mathrm{~kg} / \mathrm{m}^{3}, C_{s}=2652\right.$ $\mathrm{m} / \mathrm{s}$ ), and found that maximal pressure ratio $P_{s p} / P_{m p}$ of the secondary peak-to-main focus intensities is at most $20 \%$. However, for higher values of sound speed and devices of limited amplitude only covering a very partial area of the skull surface, we can expect much higher values of maximal pressure ratio, inducing dangerous hot spots on superficial levels of the skull.

The time-shifting and TR methods are said to limit the generation of grating lobes while improving the overall focus ability. However, especially in the domain of strong heterogeneities $\left(C_{s}>2500 \mathrm{~m} / \mathrm{s}\right)$ that strengthens hot spot intensity, the TR is not immune to the secondary pressure peaks phenomena. Indeed, as relying on phased arrays, the TR is sensitive to constructive diffraction due to the geometry of the array. Besides, as a time-delay based method, the TR leads to phase-shift between the piezotransducers of one same array therefore to an associated diffraction pattern showing secondary hot spots.

To make the HIFU system for the brain tumor treatment practicable, we have to study various aspects thereof, including the control strategy and the geometry design. In the present study, we focus on a methodological aspect on the hot spot elimination applicable to the system involving strong heterogeneity in material propertied as shown in Fig. 1. To this end, we will develop a simple algorithm as an extension of the CT-scan based TR technique. To obtain the pressure field, we will use a 3D finite difference code originally developed by Okita et al. ${ }^{(17)}$. The validity of this code has been established for various wave propagation problems. For HIFU simulations, a function of the ultrasonic wave generation by the piezotransducer has been implemented into the code. The code will be extended to execute the elimination algorithm as detailed in $\S 3$. Using high speed computers, we will perform 3D simulations of the TR problem to predict the focal point and to examine the ability of the back-propagation procedure $^{(9)}$. Then, the problem of the generation of maximal pressure secondary peaks by the heterogeneity will be studied, and the usefulness of the elimination algorithm will be investigated. Those piloting studies might provide us with the appropriate phase control strategy for array transducers experiments.

\section{Driving equations}

\subsection{Propagation equations in multicomponent field}

The present research is based on the theory of the $\mathrm{TR}^{(7)-(9)}$, and expands it to the adaptive correction of the distortions induced by the skull bone. Basic equations for simplified ideal fluid hydrodynamics ${ }^{(18)}$, which consist of the mass and momentum conservation equations with an Equation Of State (EOS) nonlinearly linking pressure to density, are numerically solved. In the practical numerical procedure for systems involving complicated geometries, 
instead of separately partitioned two velocity-pressure fields in the fluid and solid domains, it is convenient to introduce a monolithic velocity-pressure field applied to the entire computational domain. In multiphase flow simulations, one set of governing equations for the whole flow field, referred to as a one-fluid formulation ${ }^{(19)}$, is often employed. Following such an idea together with assumptions of small amplitude ultrasonic waves propagation, we use phase-averaged quantities discretized on a fixed rectangular grid. For given properties such as the density $\rho_{k}$ and the speed $C_{s, k}$ of sound of phase $k$, the mixture quantities $\rho_{m}$ and $C_{s m}$ in a computational cell are evaluated from arithmetical and harmonic means ${ }^{(20)}$, namely

$$
\begin{aligned}
& \rho_{m}=\sum_{k} f_{k} \rho_{k} . \\
& C_{s m}=\sqrt{\rho_{m}^{-1}\left\{\sum_{k} \frac{f_{k}}{\rho_{k} C_{s, k}^{2}}\right\}^{-1}},
\end{aligned}
$$

where $f_{k}$ denotes the volume fraction of the phase $k$ inside the computational cell.

In a HIFU for ESWL (Extracorporeal Shock Wave Lithotripsy) with the large pressure amplitude, the Mach number, corresponding to the ratio of the advective speed to the acoustic one, is an important parameter, and the nonlinear effect associated with the advective momentum transport plays a significant role on the shock wave formation to enable a mechanical energy supply in a very short time. By contrast, in the HIFU for the tumor heating, which requires much longer time for the temperature rise, the supplied energy rate is much lower, and the Mach number is sufficiently smaller than unity even at the focal point. In such a case, the nonlinearity in the convection involved in the Navier-Stokes equation may be omitted ${ }^{(21)}$. The dissipative effects of viscous resistance and heat conduction are neglected. Besides, the shear elasticity in the propagation media is neglected. The bulk elasticity is considered to account for the compressibility of the media. For water, the Tait equation ${ }^{(22)}$ is used as the EOS. Supposing the change in the temperature is sufficiently small, from the EOS, the density and speed sound are given as a function of the pressure. For solid, the density and the sound speed are fixed. These assumptions imply that the energy equation is redundant. Therefore, the basic equations to be considered are

$$
\begin{aligned}
& \frac{1}{\rho_{m}} \frac{\partial \rho_{m}}{\partial t}+\nabla \cdot \boldsymbol{u}=0, \\
& \rho_{m} \frac{\partial \boldsymbol{u}}{\partial t}=-\nabla p, \\
& \rho_{l}=\rho_{0}\left(\frac{p+b}{p_{0}+b}\right)^{1 / n},
\end{aligned}
$$

where $\rho_{m}$ denotes the mixture density, $p$ the monolithic pressure, $\boldsymbol{u}$ the velocity vector and the subscript $l$ a quantity of the liquid phase. The coefficients in the EOS (Eq. (6)) are $b=2.999 \times 10^{8} \mathrm{~Pa}, p_{0}=1 \times 10^{5} \mathrm{~Pa}, \rho_{0}=1 \times 10^{3} \mathrm{~kg} / \mathrm{m}^{3}$ and $n=7.15$. Note that the density-pressure relationship described by the Tait equation may be regarded as linear when the pressure is lower than a few Mega Pascal. In the present study, the wave propagation process may be approximately linear over the whole domain except near the focal point.

\subsection{Pressure generation}

Sound waves are generated from a piezotransducer (Fig. 2). To this end, in actual simulations, we treat

$$
\frac{1}{\rho_{m} C_{s m}^{2}} \frac{\partial p}{\partial t}+\nabla \cdot \boldsymbol{u}=S
$$

where $S$ is a time-dependent source given to satisfy a prescribed boundary condition in a discretized form for a piezo-liquid mixture mesh. 


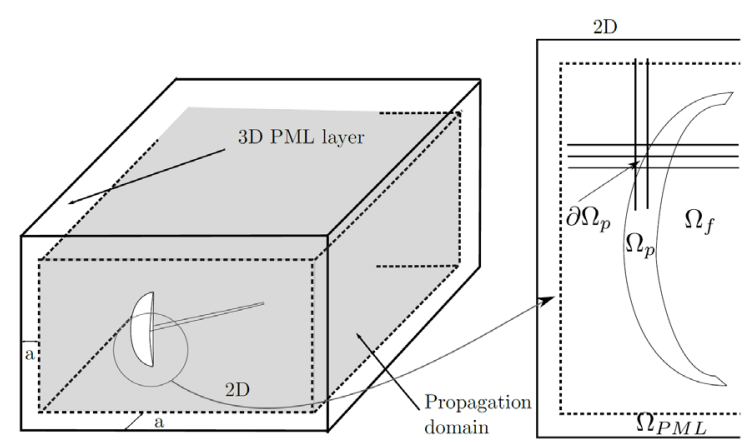

Fig. 2 A schematic of the computational domains $\Omega_{f}, \Omega_{p}$ and $\Omega_{P M L}$, which denote the domains of the fluid, piezotransducer and PML wave damper, respectively. The boundary $\partial \Omega_{p}$ is numerically treated as a fluid-piezotransducer mixture.

\section{Secondary peak elimination algorithm}

The secondary peak elimination will be attained as an extension from a simple heuristic iterative process derived from the time reversing protocol ${ }^{(9)}$. It takes advantage of the high-speed, high-precision, TR simulations involving CT-scan acquisition to predict hot spot location and deduce corrective signals. Precise numerical scans of the patient's targeted area allow for the pre-processing of the entire treatment through numerical simulations of propagation of HIFU. Ultimately, it will be possible to make use of mathematical processes such as the TR within the simulation before outputting an optimized set of signals to the real apparatus. We describe the successive steps of the algorithm below.

- First step: A usual TR process ${ }^{(8)}$ is conducted through the following two successive procedures:

i) Simulation of a wave propagation from a source located at the tumor location and recording of the pressure on the piezotransducers. The sound source is manually set at the tumor location chosen on the CT scans to mimic pointwise generator. The pressure $p_{1 a, p i e z o}(t)$ on each piezotransducer is recorded at each time step.

ii) Simulation of the backward wave propagation from the array to the tumor location. The basic equations (5), (6) and (7) are numerically solved to find the pressure field $p_{2}$. The source term $S$ in Eq. (7) is determined based on a relation $p_{2, \text { piezo }}(t)=p_{1 \text { a,piezo }}(T-t)$ at the piezo location. The resulting pressure pattern that benefices from the improvement due to the use of the TR focus control technique exhibits usually a clearer and higher focus than usual direct propagation pressure field, but is strained by secondary peaks.

- Second step: A second TR process is performed. Here, we define a maximal pressure $P$ as the maximum value in the temporal change of the numerical solution of $p$ at each grid point. The sound source is set at the location of the secondary peak, which is detected from the profile of the maximal pressure $P_{2}$ (obtained from $p_{2}$ ). The pressure $p_{1 b \text {,piezo }}$ on each piezotransducer is recorded in the same way as the first step i).

- Third step: The two sets of signals are superposed and sent back together from the piezotransducers. In the same way as the first step ii), the basic equations (5), (6) and (7) are numerically solved together with the following relation at the piezo location:

$$
p_{3, \text { piezo }}(t)=p_{1 \text { 1а,рiezo }}(T-t)+\gamma p_{1 b, \text { piezo }}(T-t-\Delta t),
$$

where $\Delta t$ and $\gamma$ are certain time delay and constant, respectively, to be determined. The resulting pressure field $p_{3}$ is given as a linear superposition of two contributions generated by two sound sources. The contribution of $p_{1 \text { a,piezo }}$ focuses back to the tumor location, whereas the contribution of $p_{1 b \text {,piezo }}$ to the secondary peak location. Optimized values of the time delay $\Delta t$ and the constant $\gamma$ are determined to minimize the superposed maxmal pressure 
$P_{3}$ (obtained from $p_{3}$ ) at the secondary peak location. The secondary peak is eventually attenuated, restoring a pressure pattern with a clearly contrasted main focus.

- Iteration steps: After the procedure of the third step is applied, another secondary peak is possibly generated because the secondary source causes extra grating lobes. The pressure profile is improved by iteration of the process of the second and third steps until elimination of all critical peaks, adding up successive sound sources at the location of the successive peaks.

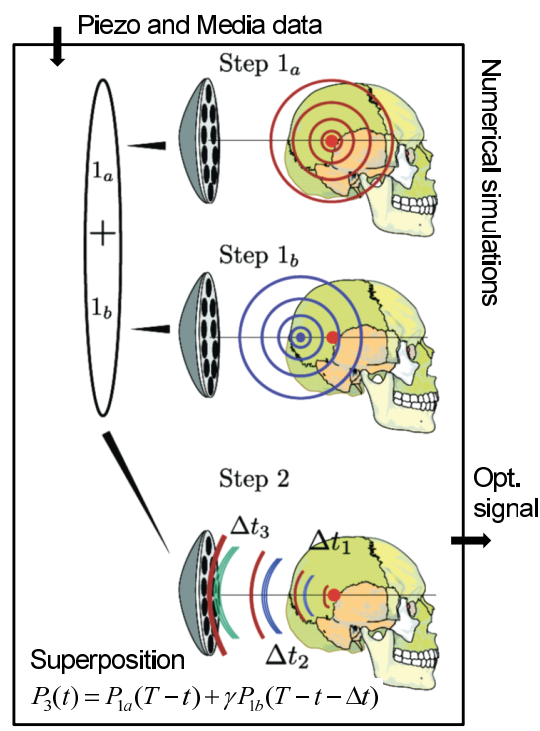

Fig. 3 A schematic of the secondary peak heuristic elimination process.

The whole procedure is schematically illustrated in Fig. 3. It should be noticed that the advocated method has demerits as well as the merits of the secondary peak elimination. The crucial demerits include problems that the iterative addition of the secondary source to the system possibly makes the envelope of the maximal pressure profile from the target wider, and/or displaces the focal position of the main peak to some extent. Taking both the merits and demerits into consideration, we will investigate how the advocated method works.

\section{Numerical method}

\subsection{General descriptions}

To cope with heterogeneity in material properties over the whole domain, the numerical method features a Finite Difference Time Domain (FDTD) computation ${ }^{(23)}$ instead of raytracing algorithms applicable to quasi-homogeneous systems. Propagation equations are discretized with a fourth-order finite-difference scheme in space ${ }^{(24)}$. The spatial derivatives and interpolation of any function $f$ from the cell center to surface (or the cell surface to center) are respectively written as:

$$
\begin{aligned}
\left.\partial_{x} f\right|_{i+1 / 2} & =\frac{27\left(\left.f\right|_{i+1}-\left.f\right|_{i}\right)-\left(\left.f\right|_{i+2}-\left.f\right|_{i-1}\right)}{24(\Delta x)}, \\
\left.f\right|_{i+1 / 2} & =\frac{9\left(\left.f\right|_{i+1}+\left.f\right|_{i}\right)-\left(\left.f\right|_{i+2}+\left.f\right|_{i-1}\right)}{8},
\end{aligned}
$$

where $i$ stands for the node number, and $\Delta x$ denotes the grid width. These expressions ensure that the transported quantities and their second moments are conserved in a discretized space. To obtain a viable compromise between a standard value $(\sim 1 \mathrm{MHz})$ in therapeutic applications and the available numerical resources, we choose the frequency to be $500 \mathrm{kHz}$. The grid width is set to $\Delta x=0.5 \mathrm{~mm}$, and thus the wavelength is resolved by at least six grid points.

The non-reflecting boundary condition on the limits of the computational domain is implemented by use of a Perfectly Matched Layer (PML) ${ }^{(25)}$, to provide an good absorbing layer 
mimicking infinite media (Fig. 2). The width of PML is set to $a=15 \Delta x$, which is sufficiently long to reduce reflection on the numerical boundary to undetectable values. Hence, the width of the surrounding domain is $L+2 a$, where $L$ is the width used for the analysis.

A numerical domain is spatially decomposed into 64 subdomains for parallel computing over 64 processors on the RIKEN super-cluster quest. Each subdomain is allocated to each processor, and a MPI library is implemented in the code for the data communication between the processors.

Post-processing as well as pre-processing of output or input volume data and other discrete set of values are performed with the RIKEN-made software Visio ${ }^{(C)}$ and Vxgen ${ }^{\mathcal{C}}$. The $3 \mathrm{D}$ visualization of volume fraction uses shader rendition.

\subsection{Implementations of geometry data}

To implement the geometry information into the simulation code, we employ two methods for 1) sharp interface data based on a blueprint and for 2) diffuse interface data converted from a medical image as explained below:

1) Piezotransducers and Homogeneous plate: Objects of simple geometry and homogeneous composition can be directly hard-coded in the source program or imported from CAD files through the Pre-processing tool Vxgen ${ }^{C}$. From given surface data of the piezotransducer array or the homogeneous plate, a signed distance function ${ }^{(26)(17)}$ can be directly computed on each grid point. Therefrom, the local volume fraction, which is regarded as a smoothed Heaviside function $^{(27)}$ with a grid scale smoothness, is identified to describe the geometry information over the whole computational domain.

2) Human skull volume data: The geometry and properties of the human skull are available in a voxel data form, including the material volume fraction, discretized on a $600 \times 400 \times 400$ cells. Unlike a number of other methods, the data available in CT scans also include a description of skin and brain areas. It is therefore possible to take into account the distortion induced by the propagation into those areas. The original CT scan data are given in Hounsfield Units such as:

$$
H=1000 \times \frac{\mu-\mu_{\text {water }}}{\mu_{\text {bone }}-\mu_{\text {water }}},
$$

where $\mu, \mu_{\text {water }}$ and $\mu_{\text {bone }}$ defined respectively the luminosity attenuation coefficient of the current cell, water and bone. We use then the relation proposed by Fink et al. ${ }^{(7)}$ to link porosity and Hounsfield units values:

$$
\Phi=1-\frac{H}{1000},
$$

where $\Phi$ is the porosity, that is the volume fraction in the current cell. Note that the spatial variation of $\Phi$ inside the skull is considered in the simulation to capture the multiple reflexion of the sound waves over the diverse wall of the inner cavity.

The presence of the bone or the plate in each computational cell is expressed in a mixture form of the density $\rho_{m}$ and the sound speed $C_{s m}$ given by Eqs. (2) and (3).

\section{Application to a homogeneous solid plate}

To illuminate the fundamental aspect of the problem and to demonstrate the effectiveness of the secondary peak elimination method advocated in $\S 3$, a simple system involving a flat plate will be considered here. The first application examined here will be the direct propagation of HIFU through a flat plate of thickness $d=0.5 \mathrm{~cm}$, homogeneous density $\rho=1700$ $\mathrm{kg} / \mathrm{m}^{3}$ and bulk modulus $K=15.0 \mathrm{GPa}$. The corresponding sound speed is $C_{s} \sim 3000 \mathrm{~m} / \mathrm{s}$. A quasi-continuously phased piezotransducer with a $8 \mathrm{~cm}$ curvature comprised of 227 elements is set at a $6 \mathrm{~cm}$ distance from plate (see Fig. 4). Each piezotransducer radius is of millimetric scale $^{(9)}$. To show the relevance of such a hard material to the hot spot intensity, the HIFU simulations are performed at a fixed frequency of $500 \mathrm{kHz}$ with varying the sound speed $C_{s}$ 


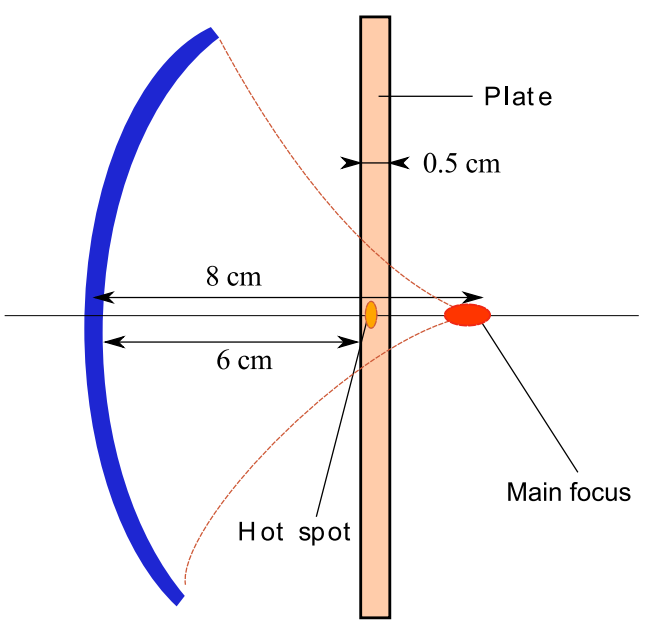

Fig. 4 A schematic figure of a HIFU system involving a flat plate.

of the plate. Further, the simulations with density and bulk modulus profiles identical to those of a skull bone with an approximate $5 \mathrm{~mm}$ occipital thickness ${ }^{(28)}$ (Fig. 5) are also carried out. Figure 6 shows a maximal pressure ratio $P_{s p} / P_{m p}$ (here, $P_{s p}$ denotes the highest maximal pressure among the secondary spots, and $P_{m p}$ the maximal pressure at the main focal point) as a function of $C_{s}$. The ratio $P_{s p} / P_{m p}$ is positively correlated with $C_{s}$, and the secondary pressure $P_{s p}$ is likely to be even higher than $P_{m p}$ for the relatively high $C_{s}$, revealing the danger of those hot spots whilst dealing with high amplitude pressure value such as medical treatments. Propagation through a homogeneous plate with sharp edges presents even higher values of secondary peaks maximal pressure.

Those conditions constitute excellent cases for algorithm testing. The TR and secondary peak elimination algorithm is applied to the system involving the above-mentioned homogeneous plate at the sound speed of $C_{s}=2900 \mathrm{~m} / \mathrm{s}$. To show the effects of the TR and peak elimination procedures, Fig. 7 reports the axial profiles of the maximal pressures. The direct propagation of HIFU uniformly pulsed by the piezotransducer reveals a strong displacement of the main focal point located at $D=-0.6 \mathrm{~cm}$ (here, $D$ denotes the distance to the target equivalent to the abscissa of Fig. 7), and the generation of a secondary peak at $D=-2.0$ $\mathrm{cm}$, corresponding to the location close to the interface of the plate (see Fig. 4). Notably, the intensity of the secondary peak is higher than that of the main peak (namely, $P_{s p} / P_{m p}>1$ ) as already indicated in Fig. 6. The simple TR procedure is confirmed to effectively control the main focal point to shift toward the target, and also to intensify its maximal pressure. However, the secondary peak intensity at $D=-2.0 \mathrm{~cm}$ is still higher than that of the main peak, indicating that the simple TR procedure is not much useful for attenuating the undesirable hot spot intensity. Upon setting up a second source of amplitude $0.12 A$ (here, $A$ is the amplitude of the original primary source) at $D=-2.0 \mathrm{~cm}$, the elimination procedure based on the second and third steps in $\S 3$ is applied. As shown in Fig. 7 , the intensity at $D=-2.0$ $\mathrm{cm}$ is considerably reduced, indicating that the procedure decreases the secondary peak to more acceptable values. However, there exists another strong surge at $D=-3.9 \mathrm{~cm}$, whose magnitude is comparable to that of the main focus, because of the grating lobes stemming from the second source. Furthermore, the main peak location undergoes a shift of $-0.2 \mathrm{~cm}$, where a redistribution of energy and a heat deposition would be noticeable. Once again, as the iteration step in $\S 3$, the elimination procedure is applied with the third source at $D=-3.9$ $\mathrm{cm}$. As a negative signature of the iterative method, each step of the elimination procedure is found to entail a negative shift of the main peak since a high value of secondary source amplitude is imposed in order to compensate the extreme value of maximal pressure on the grating lobe. Furthermore, due to the iterated procedure, the peak intensity at $D=-2.0 \mathrm{~cm}$ 


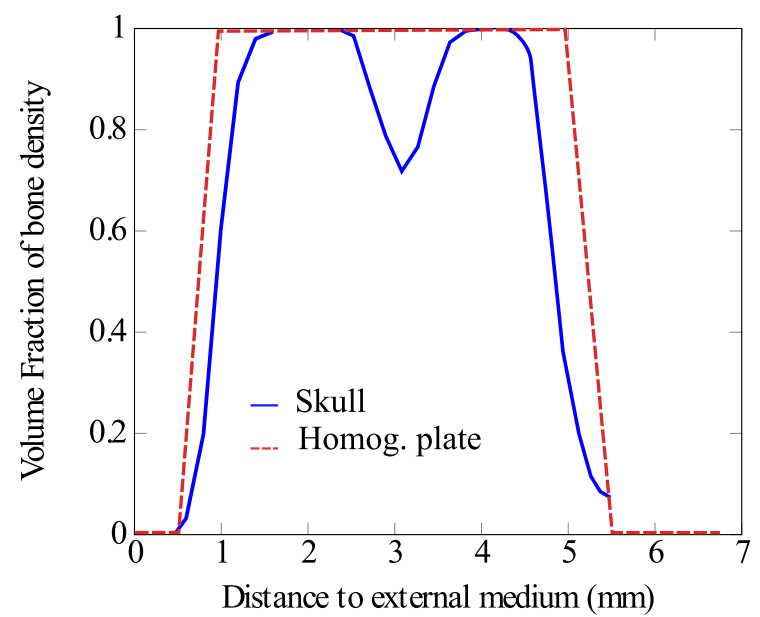

Fig. 5 Typical Volume fraction profiles of bone material close to the skull and a homogeneous equivalent.

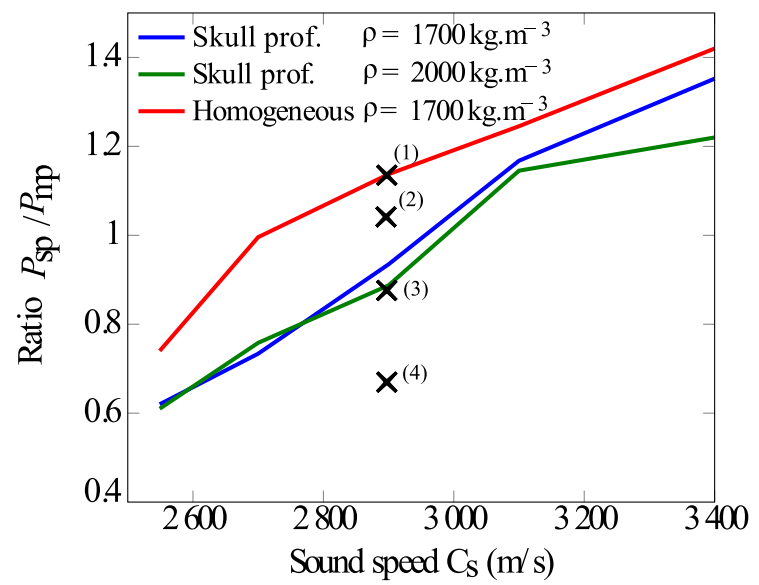

Fig. 6 The maximal pressure ratio $P_{s p} / P_{m p}$ versus the sound speed $C_{s}$ in various plate cases (direct propagation). The crosses indicate the evolution of the pressure ratio for (1) Direct propagation, (2) Simple TR, (3) TR + secondary peak elimination, and (4) TR + iterated peak elimination.

becomes slightly higher, and the decay with distance from the main peak becomes slower for $D \gtrsim 1 \mathrm{~cm}$. The iterative procedure should be terminated when the highest intensity among all the lobes falls below a certain level. Nevertheless, as shown in Fig. 7, the maximal pressure ratio $P_{s p} / P_{m p}$ is considerably reduced to 0.67 or less over the entire range, and therefore the iterative method is concluded to be useful for providing a desirable profile in the maximal pressure pattern, which is attained by the combined three independent signals recorded from the three sound sources. The usefulness is also ascertained quantitatively from the secondary peak attenuation in Fig. 6, which reports the evolution of the pressure ratio $P_{s p} / P_{m p}$ indicated by the symbols $\times$.

\section{Application to cranial tumor elimination}

The case of HIFU treatment of deep-seated brain tumors is one of the most promising fields for the application of medical ultrasound. Overcoming the focus displacement due to the distortion induced by the skull bone remains a major challenge. Although the TR procedure was found to be effective for a monkey skull ${ }^{(9)}$, the human skull is much thicker and therefore prone to high levels of pressure field distortion. Water-cooling of the bone hot spots remains the common law to avoid skin burns and friction effects on the bone. A totally non-invasive treatment would require to take care of the superficial hot spots. 


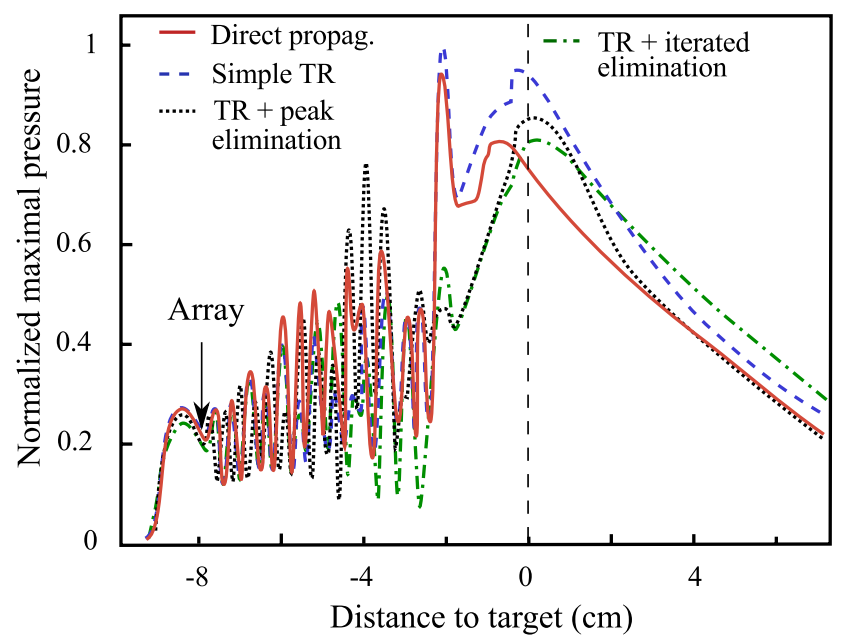

Fig. 7 Maximal pressure profile along the acoustical axis.

In this matter, we perform simulations using a numeric phantom of human skull, which is the same as that demonstrated in Fig. 1. The voxel-based skull as well as the phased array are expressed over the computational domain in a mixture form as explained in \$4.2. Typical values for maximal bulk modulus and density in the skull bones depend on the location, but the average values of trabecular bone in occipital or frontal areas were taken as $K=15 \mathrm{GPa}$ and $\rho=2000 \mathrm{~kg} / \mathrm{m}^{3}$. Although the bulk modulus chosen here is lower than that of a normal skull bone $(\sim 20 \mathrm{GPa})^{(29)}$ due to the limitation of the available numerical resources, the resulting sound speed $\left(C_{s}=2900 \mathrm{~m} / \mathrm{s}\right)$ is high enough to generate the hot spot. Therefore, we may discuss the effectiveness of the secondary peak elimination method applied to the practical system.

The ultrasonic device itself is a cap-shaped phased array transducer with a $6.5 \mathrm{~cm}$ curvature and $6 \mathrm{~cm}$ envergure as illustrated in Fig. 8, though a larger focused ultrasound array transducer, which can cover a wide portion of the skull cavity, enables to reduce the formation of hot spots by decreasing the acoustic energy penetrating the skull from each transducer elements. The array is comprised of 227 piezotransducer elements, of which each radius is of millimetric scale ${ }^{(9)}$. During the first step of the TR procedure, a sound source with a 500 $\mathrm{kHz}$ sine signal is put at a target, corresponding to the centroid of the brain tumor located at a $2.5 \mathrm{~cm}$ distance from the frontal lobe outer wall. The heterogeneous structure of the trabecular bone is typically of millimetric scale ${ }^{(29)}$. Tiny-scale phenomena associated with the bone heterogeneity cannot be faithfully resolved in the present simulation. However, it is of primary importance to investigate how the secondary peak elimination method modifies the focal point displacement and the hot spot generation caused by the reflection and refraction of ultrasound. Such a wavelength-scale phenomenon can be reasonably captured under the present simulation conditions.

Figure 9 reports the cross-sectional views of the maximal pressure patterns normalized by the maximum value. Figure 9(a) exhibits a very distorted pressure pattern. It should be noted that the main focus shifts out of the acoustical plane. Besides, a strong secondary peak is generated close to the bone. As shown in Fig. 9(b), the simple TR procedure is effective for improving the relative intensity of the main peak. The position of the main peak in the acoustical plane is three-dimensionally corrected. Although the secondary peak is attenuated, it remains at the same location. The secondary peak elimination procedure is applied with the second source at the secondary peak. As shown in Fig. 9(c), the procedure relatively intensifies the main focus. Note that unlike the results with the homogeneous solid flat plate shown in Fig. 7, the secondary peak is sufficiently attenuated only with a single iteration, and another secondary peak due to the grating lobes induced by the second source does not clearly 


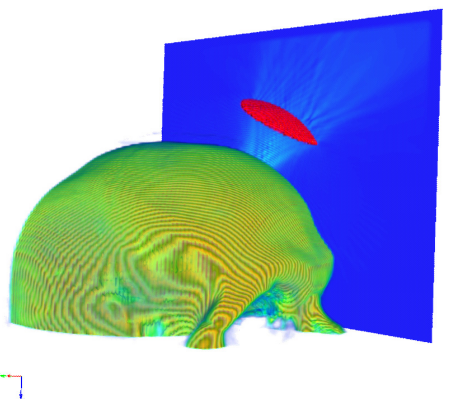

(a) Front view

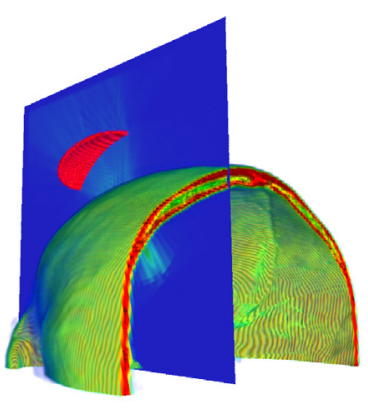

(b) Back view

Fig. 8 The computational domain involving a human skull and a cap-shaped phased array, which are numerically expressed as a voxel-based geometry. The gray scale display in the cross-sectional plane shows a typical simulation result of pressure intensity distributions.

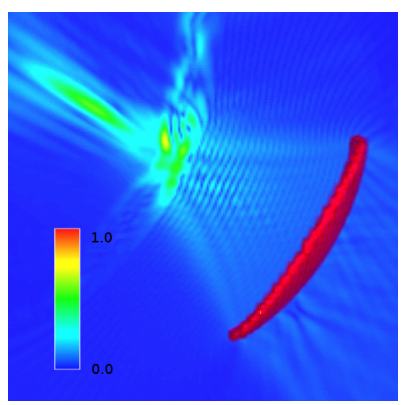

(a) Direct propagation

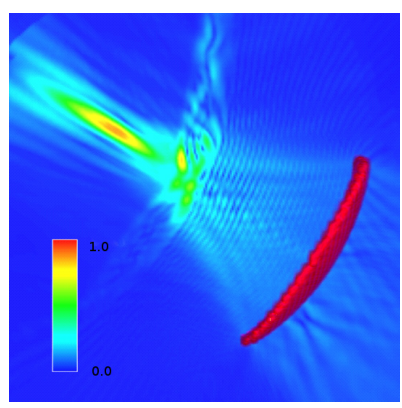

(b) Simple TR

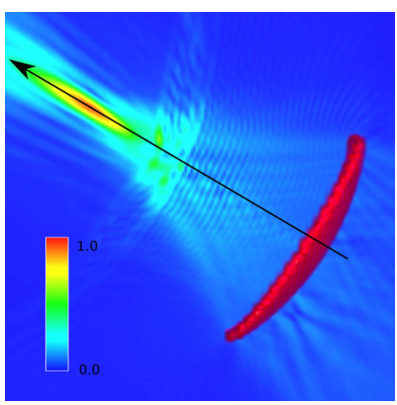

(c) TR + secondary peak elimination (1 iteration)

Fig. 9 Cross-sections of maximal pressure on an acoustical plane.

form.

To quantitatively show the effects of the TR and peak elimination procedures, Fig. 10 reports the normalized maximal pressure along an axis, which connects the target and the center of the transducer, as drawn in Fig. 9(c). Moreover, the distance between the main focus and the target, and the maximal pressure ratio $P_{s p} / P_{m p}$ are evaluated from the threedimensional simulated data, and summarized in Table 1. It should be noticed that since the main and secondary peaks are not always located on the axis, the pressure ratio $P_{s p} / P_{m p}$ in Table 1 differs slightly from that evaluated from the axial profile in Fig. 10. Even in the case of the direct propagation with no TR or peak elimination procedure, the displacement of the main focus is not clearly seen from Fig. 10. The main focus is distorted almost on a perpendicular acoustical plane. The intensity of the secondary peak at $D=-2.0 \mathrm{~cm}$ is even higher than that of the main peak as shown in Fig. 10, that is reflected on the value $P_{s p} / P_{m p}>1$ in Table 1 . When the simple TR procedure is applied, the main focus is redirected towards the acoustical axis, and thus the distance between the main focus and the target is shortened from $3.20 \mathrm{~mm}$ to $0.86 \mathrm{~mm}$ (Table 1). Although the secondary peak at $D=-2.0 \mathrm{~cm}$ is noticeably corrected, its peaky profile is still obvious. When the second peak elimination procedure is applied, the second peak intensity at $D=-2.0 \mathrm{~cm}$ and thus the grating lobe intensity over the whole domain are decreased to more acceptable values as confirmed in the profile of the pressure (Fig. 10) and in the reduction in $P_{s p} / P_{m p}$ (Table 1). The distance between the main focus and the target becomes slightly longer due to the elimination procedure as shown in Table 1. However, this level of the displacement is much smaller than a typical length scale of the ablation region in the HIFU therapy being a few centimeters. Note that in the more practical 


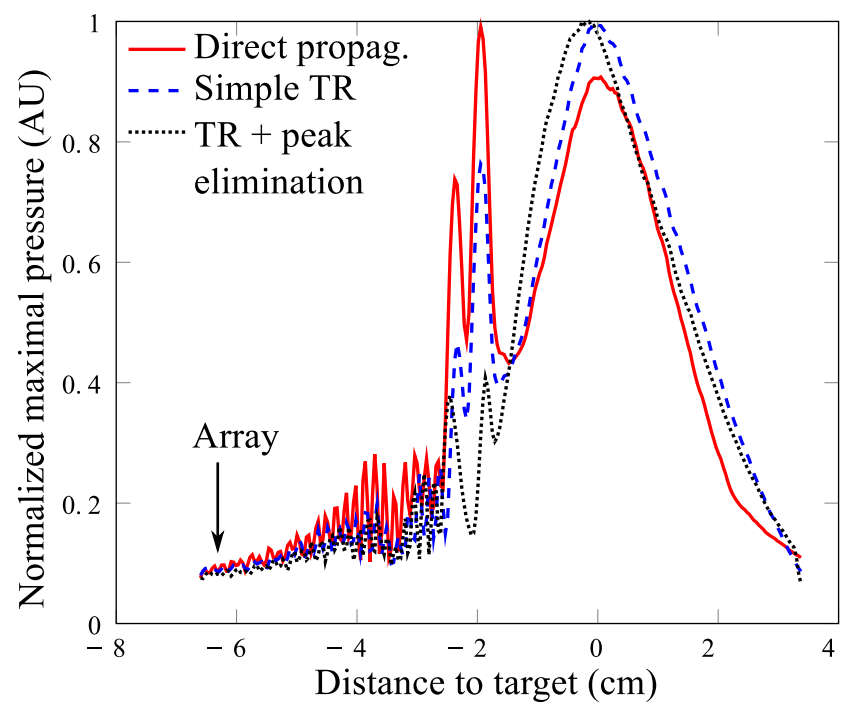

Fig. 10 The normalized maximal pressure profile along the acoustical axis. The array location corresponds to $-6 \mathrm{~cm}$ in the abscissa.

situation with the higher frequency of $\sim 1 \mathrm{MHz}$, the displacement due to the elimination procedure would be further shorter due to the shorter wavelength.

Table 1 Evolution of focus location and pressure ratio with the TR procedure and secondary peak elimination

\begin{tabular}{lcc}
\hline Case & Dist. from main focus to target $(\mathrm{mm})$ & Max. pressure ratio $P_{s p} / P_{m p}$ \\
\hline Direct propagation & 3.20 & 1.14 \\
Simple TR & 0.86 & 0.72 \\
TR + sec. peak elim. & 1.22 & 0.33 \\
\hline
\end{tabular}

\section{Conclusion}

The non-invasive, CT-based Time Reversal (TR) process $^{(7)-(9)}$ has been extended to the obliteration of the superficial hot spots close to the skull. A numerical, heuristic, and iterative procedure has been developed to correct the maximal pressure profile in numerical simulations of HIFU propagation. The numerical simulations with a plate and a human skull showed that although the secondary peak elimination procedure is likely to make the envelope of the maximal pressure profile from the target wider, it can effectively attenuates the undesirable hot spot intensity from severe overheating to benign values. A treatment of thicker or highly reflective areas would be possible through iteration of the procedure. It is feasible to combine CT-scans, the TR induced propagation and the secondary peak elimination procedure, and to numerically prepare a set of optimized driving signals for experimental apparatus. The use of the peak elimination procedure could pave the way to a fully non-invasive procedure avoiding the overheating of the skull without using increased penetration area.

It should be noticed that after the first application of the secondary peak elimination procedure, another hot spot was intensified as described in $\S 5$. This result implies a possibility that the iterative procedure fails to attenuate the every lobe intensity below a threshold in some cases (which may be dependent upon e.g. the bone thickness and the tumor location). Even though we have not encountered such a problem, further parametric studies will be needed to elucidate the domain of the applicability of the elimination method.

It is essential to experimentally perform validation tests in order to evaluate whether the proposed procedure works and guarantees the non-invasiveness in reality. An ex vivo experiment is the ongoing subject of the present authors using a HIFU system with array piezotransducers. 


\section{Acknowledgments}

The authors thank Tetsuya Kanagawa for fruitful discussion, and the referees for their useful comments and suggestions. This research was supported by Research and Development of the Next-Generation Integrated Simulation of Living Matter, a part of the Development and Use of the Next- Generation Supercomputer Project of the Ministry of Education, Culture, Sports, Science and Technology (MEXT), and by the Grant-in-Aid for Young Scientist (B) (No.21760120) of MEXT. NL was financially supported by the Japanese MEXT.

\section{References}

( 1 ) Clement, G.T., White, J. and Hynynen, K., "Investigation of a large-area phased array for focused ultrasound surgery through the skull”, Phys. Med. Biol., Vol. 45 (2000), pp. 1071-1083.

( 2 ) Sun, J. and Hynynen, K., "Focusing of therapeutic ultrasound through a human skull", J. Acoust. Soc. Am., Vol. 104 (1998), pp. 1705-1715.

( 3 ) Sun, J. and Hynynen, K., "The potential of transskull ultrasound therapy and surgery using the maximum available skull surface area", J. Acoust. Soc. Am., Vol. 105, (1999), pp. 2519-2527.

( 4 ) Fink, M., "Time reversal of ultrasonic fields. Part I: Basic principles", IEEE Trans. Ultrason. Ferroelectr. Freq. Control, Vol. 39 (1992), pp. 555-566.

( 5 ) Fink, M., Cassereau, D., Derode, A. Prada, C., Roux, P., Tanter, M., Thomas, J.L. and Wu, F., “Time-reversed acoustics", Rep. Prog. Phys., Vol. 63 (2000), pp. 1933-1995.

( 6 ) Cassereau, D. and Fink, M., "Time-reversal of ultrasonic fields. III. Theory of the closed time-reversal cavity”, IEEE Trans. Ultrason., Ferroelectr. Freq. Control, Vol. 39 (1992), pp. 579-592.

( 7 ) Fink, M., Montaldo, G. and Tanter, M., "Time Reversal Acoustics in Biomedical and Engineering”, Annu. Rev. Biomed. Engrg., Vol. 5 (2003), pp. 465-497.

( 8 ) Aubry, J.F., Tanter, M., Pernot, M., Thomas, J.L. and Fink, M., "Experimental demonstration of noninvasive transskull adaptive focusing based on prior computed tomography scans", J. Acoust. Soc. Am., Vol. 113 (2003), pp. 84-93.

( 9 ) Marquet, F., Pernot, M., Aubry, J.F., Montaldo, G., Marsac, L., Tanter, M. and Fink, M., "Non-invasive transcranial ultrasound therapy based on a 3D CT scan", Phys. Med. Biol., Vol. 54 (2009), pp. 2597-2613.

(10) Cheston, T.C. and Frank, J., 'Phased Array Radar Antenna Radar Handbook', Chap. 7, (1990), pp. 1-8.

(11) Anderson, B.E., Hughes, W.J. and Hambric, S.A., "Grating lobe reduction in transducer arrays through structural filtering of supercritical plates”, J. Acoust. Soc. Am., Vol. 126 (2009), pp. 612-619.

(12) Pompei, F.J. and Wooh, S.C., "Phased array element shapes for suppressing grating lobes", J. Acoust. Soc. Am., Vol. 111 (2002), pp. 2040-2048.

(13) Hildebrand, B.P. and Posakony, G.J., "Method and apparatus for energizing an array of acoustic transducers to eliminate grating lobes", Dec. 18 (1979), US Patent 4,179,683.

(14) Dupenloup, F., Chapelon, J.Y., Cathignol, D.J. and Sapozhnikov, O.A., "Reduction of the grating lobes of annular arrays used in focusedultrasound surgery", IEEE Trans. Ultrason. Ferroelectr. Freq. Control, Vol. 43 (1996), pp. 991-998.

(15) Gavrilov, L.R. and Hand, J.W., "Two-dimensional phased arrays for surgery: movement of a single focus", Acoust. Phys., Vol. 46 (2000), pp. 390-399.

(16) Hutchinson, E.B., Buchanan, M.T. and Hynynen, K., "Design and optimization of an aperiodic ultrasound phased array for intracavitary prostate thermal therapies", Med. Phys., Vol. 23 (1996), pp. 767-776.

(17) Okita, K., Ono, K., Takagi, S. and Matsumoto, Y., "Development of high intensity focused ultrasound simulator for large-scale computing”, Int. J. Numer. Meth. Fluids, Vol. 65 (2011), pp. 43-66. 
(18) Ginter, S., Liebler, M., Steiger, E., Dreyer, T. and Riedlinger, R.E., "Full-wave modeling of therapeutic ultrasound: nonlinear ultrasound propagation in ideal fluids", J. Acoust. Soc. Am., Vol. 111 (2002), pp. 2049-2059.

(19) Tryggvason, G., Sussman, M. and Hussaini, M.Y., "Immersed boundary methods for fluid interfaces", in Prosperetti and Tryggvason, 'Computational methods for multiphase flow', Chap. 3, Cambridge University Press (2007).

(20) Drew, D.A. and Passman, S.L. 'Theory of Multi-Component Fluid', Chap. 22, SpringerVerlag, New York, (1999).

(21) Landau, L.D. and Lifshitz, E.M., 'Fluid Mechanics', second edition, Chap. 8, Butterworth-Heinemann, Oxford, (1987).

(22) Tait, P.G, "On the properties of water" in 'Physics and Chemistry of the Voyage of H.M.S Challenger, HMSO, London', (1888).

(23) Taflove, A., "Application of the finite-difference time-domain method to sinusoidal steady-state electromagnetic-penetration problems", IEEE Trans. Electromagnetic Compatibility, Vol. EMC-22 (1980), pp. 191-202.

(24) Kajishima, T., Takiguchi, S., Hamasaki, H. and Miyake, Y., "Turbulence structure of particule-laden flow in a vertical plane channel due to vortex shedding", JSME INT. J. Ser. B, Vol. 44 (2001), pp. 526-535.

(25) Berenger, J.P., "Three-dimensional perfectly matched layer for the absorption of electromagnetic waves", J. Comput. Phys., Vol. 127 (1996), pp. 363-379.

(26) Osher, S. and Fedkiw, R. 'Level set methods and dynamic implicit surface', Chap. 1, Springer-Verlag, New York, (2003).

(27) Peskin, C.S., "The immersed boundary method", Acta Numerica, Vol. 11 (2003), pp. 479-517.

(28) Adeloye, A., Kattan, K.R. and Silverman, F.N., "Thickness of the normal skull in the American blacks and whites”, Am. J. Phys. Anthropology, Vol. 43 (2005), pp. 23-30.

(29) International Commission on Radiation Units and Measurements Tissue substitutes, phantoms and computational modelling in medical ultrasound, ICRU Rep., Vol. 61 (1998). 